\title{
Cyclooxygenase-2 expression in keratocystic odontogenic tumour decreased following decompression
}

\author{
JING WANG ${ }^{1}$, XIAOMIN ZHANG ${ }^{1}$, XU DING $^{1}$, SHUZHONG XING $^{1}$, HUAIQI LI $^{1}$, \\ WEI ZHANG ${ }^{2}$, LIZHEN WANG ${ }^{3}$ and HEMING WU ${ }^{1}$ \\ ${ }^{1}$ Institute of Stomatology, Department of Oral and Maxillofacial Surgery, School of Stomatology, Nanjing Medical University; \\ ${ }^{2}$ Division of Pathology, Jiangsu Stomatological Hospital, Nanjing, Jiangsu 210029; ${ }^{3}$ Division of Oral Pathology, \\ Shanghai Ninth People's Hospital, Shanghai 200011, P.R. China
}

Received April 15, 2013; Accepted July 3, 2013

DOI: $10.3892 / \mathrm{mco} .2013 .169$

\begin{abstract}
Marsupialisation or decompression is frequently performed as a conservative therapy for keratocystic odontogenic tumours (KCOTs). Positive cyclooxygenase-2 (COX-2) expression in the epithelium of KCOTs was recently reported and may be associated with neoplastic invasion and progression. The aim of the present study was to investigate the change in COX-2 expression in the KCOT epithelium following decompression. In this study, 16 pairs of KCOT biopsy specimens obtained during decompression or enucleation were collected and analysed. Formalin-fixed, paraffin-embedded blocks were sectioned and immunohistochemically investigated using anti-COX-2 antibody. The molecular expression was semiquantitatively evaluated as follows: 0 , negative; 1 , weakly to moderately positive; and 2 , strongly positive. In the samples obtained prior to decompression, the positive staining for COX-2 was immunolocalised to the cell membrane and the cytoplasm, it involved the full thickness of the epithelium and 15 of the 16 specimens (93.8\%) exhibited mild to strong positivity. As regards the samples obtained following decompression, only 3 of the 16 specimens (18.8\%) exhibited a mild positivity. The expression levels of COX-2 were significantly decreased following decompression $(\mathrm{P}<0.05)$. It may be concluded that loss or a significant reduction of COX-2 expression is associated with decompression in KCOTs. However, large-scale studies are required to verify these results and improve our knowledge
\end{abstract}

Correspondence to: Dr Heming Wu, Jiangsu Stomatological Hospital, 136 Hanzhong Road, Gulou, Nanjing, Jiangsu 210029, P.R. China

E-mail:whmnj2013@163.com

Dr Lizhen Wang, Division of Oral Pathology, Shanghai Ninth People's Hospital, 639 Zhizaoju Road, Shanghai 200011, P.R. China Email: wanglizhen9@126.com

Key words: immunohistochemistry, keratocystic odontogenic tumour, decompression, cyclooxygenase-2 of the possible involvement of COX-2 in the pathogenic mechanism underlying the development of KCOTs.

\section{Introduction}

Unlike other odontogenic cystic lesions, the odontogenic keratocyst (OKC) exhibits an intrinsic growth potential and a marked ability to destroy bone $(1,2)$. Clinically, following conventional treatment such as enucleation, OKC exhibits a high tendency for recurrence $(1,3)$. According to the 2005 World Health Organization classification, OKC was reclassified as a benign neoplasm, referred to as keratocystic odontogenic tumour (KCOT) $(2,3)$.

In the field of molecular biology, several immunohistochemical studies investigated the activity of the KCOT epithelium by using various markers of proliferation and apoptosis (4-7). The results of those studies demonstrated that p53 (4), PCNA (5) and Ki-67 (6) were more strongly expressed in the KCOT epithelium compared to other types of odontogenic cysts. Thus, it was concluded that the KCOT epithelium was highly proliferative, which may explain the high recurrence rate of KCOT and suggests that KCOTs have a different biological origin (7).

Recently, marsupialisation or decompression combined with two-stage enucleation was proven to be an effective treatment for large KCOTs (3). According to data reported by Ninomiya et al (8), the size of the KCOT lesions was significantly decreased following decompression. To improve the outcome of decompression, investigation of the underlying molecular mechanisms of this type of therapy is required.

Cyclooxygenase-2 (COX-2) levels were found to be elevated in various types of tumours, such as esophageal and head and neck tumours $(9,10)$. Accumulating evidence suggests that the overexpression of COX-2 is involved in tumour growth and spread by interfering with different biological processes, such as cell proliferation, adhesion, immune-surveillance, apoptosis and angiogenesis (11). The regulation of COX-2 expression is crucial for prostaglandin E2 (PGE2) synthesis $(11,12)$. The upregulation of COX-2 may increase the synthesis of prostaglandins (PGs), thereby promoting cell proliferation and angiogenesis and inhibiting immune-surveillance $(12,13)$. Recently, a study conducted by Mendes et al (7) demonstrated 
a mild to strong expression of COX-2 in the KCOT epithelium and suggested that COX-2 is an important marker involved in the biological behavior of KCOTs.

To the best of our knowledge, no studies are currently available on the mechanism of change in COX-2 expression following decompression. This is the first study to demonstrate the significant downregulation of COX-2 expression in KCOT following decompression, through the immunohistochemical investigation of 16 cases in the present series.

\section{Materials and methods}

Patients. This series comprised 16 patients with histologically confirmed KCOT at Jiangsu Stomatological Hospital and Shanghai Ninth People's Hospital. Recurrent cases or those associated with nevoid basal cell carcinoma syndrome were excluded from this study. The patients underwent decompression surgery followed by two-stage enucleation. The clinical information of the patients is provided in Table I. Postoperative follow-up comprised clinical and radiographic examinations from January, 2004 to September, 2012. The average duration of draining and irrigation prior to the two-stage surgery was 19.5 months (range, 6.5-44 months). Paraffin specimens of the tissue samples obtained at the time of decompression and enucleation were collected from the Divisions of Oral Pathology of Jiangsu Stomatological Hospital and Shanghai Ninth People's Hospital. All the patients provided signed informed consent prior to enrollment and the study was approved by the Ethics Committees of the Shanghai Ninth People's Hospital and the Jiangsu Stomatological Hospital.

Immunohistochemical evaluation. Each of the 16 pairs of formalin-fixed, paraffin-embedded samples was cut continuously into two 4- $\mu \mathrm{m}$ sections and mounted on glass microscope slides. One section from each pair was prepared for immunohistochemical COX-2 analysis and the other section was used as a negative control by replacing the anti-COX-2 primary antibody with phosphate-buffered saline. Briefly, the deparaffinised sections were immersed in absolute methanol containing $0.3 \% \mathrm{H}_{2} \mathrm{O}_{2}$ for $15 \mathrm{~min}$ at room temperature to block endogenous peroxidase activity. After washing, the sections were immersed in $0.01 \mathrm{M}$ citrate buffer ( $\mathrm{pH}$ 6.0) and heated in a microwave oven at $95^{\circ} \mathrm{C}$ for $5 \mathrm{~min}$. A properly diluted (1:100) rabbit monoclonal anti-human COX-2 antibody (product code, BS1076; Bioworld Technology, Inc., St. Louis Park, MN, USA) was then applied to the sections at $4^{\circ} \mathrm{C}$ overnight, followed by a prediluted anti-mouse IgG antibody conjugated with peroxidase (Nichirei, Tokyo, Japan) for $1 \mathrm{~h}$ at room temperature. The sections were immersed in $0.05 \%$ 3,3'-diaminobenzidine tetrahydrochloride in $0.05 \mathrm{M}$ Tris- $\mathrm{HCl}$ buffer ( $\mathrm{pH} 8.5$ ) containing $0.01 \% \mathrm{H}_{2} \mathrm{O}_{2}$ for $8 \mathrm{~min}$ and then counterstained with haematoxylin.

Immunohistochemical evaluation. Immunohistochemical reactivity for COX-2 was evaluated using a semi-quantitative detection method as previously described (7) and classified into three groups according to the intensity score as follows: 0 , negative; 1 , weakly to moderately positive; and 2 , strongly positive. The evaluation was performed by two experienced pathologists who were blinded to the clinical data.
Statistical analysis. The Student's paired t-test was used to evaluate the differentiation of COX-2 immunoreactivity in KCOTs at the time of enucleation compared to that at the time of decompression. SPSS 17.0 software (SPSS Inc., Chicago, IL, USA) was used for statistical analysis. Specific data are provided in Table I. $\mathrm{P}<0.05$ was considered to indicate a statistically significant difference.

\section{Results}

In our study, the collection of samples obtained at the time of decompression were referred to as group I and those obtained at the time of two-stage enucleation as group II. The immunohistochemical staining pattern of COX-2 in KCOT presented as membranous and cytoplasmic. In group I, positive COX-2 immunostaining was present in 15 of the 16 samples $(93 / 8 \%)$ and involved the full thickness of the epithelium, with 6 specimens exhibiting strong expression and 9 mild positivity (Fig. 1A-C). In group II, 4 of the 16 cases exhibited either mild (Fig. 1D) or negative COX-2 expression, consistent with their corresponding counterparts in group I. In the remaining 12 cases, COX-2 expression was significantly decreased (negative COX-2 expression).

The mean of intensity scores in group I was $1.31 \pm 0.60$, whereas the mean of intensity scores in group II was $0.25 \pm 0.45$. The P-value of group I vs. group II in COX-2 staining intensity was 0.00004 . The statistical analysis demonstrated a significant decrease in COX-2 expression of group II compared to that of group I $(\mathrm{P}<0.05)$.

\section{Discussion}

Marsupialisation or decompression is frequently performed as a conservative therapy for large KCOTs, since this therapy is considered beneficial in reducing the size and the recurrence rate of the tumour (14). Nakamura et al (15) reported that $96 \%$ of the cases included in their study exhibited a cystic reduction of $\geq 50 \%$. Histologically, the typical characteristics of KCOT were eliminated following this type of therapy, with the lesion assuming the form of hyperplastic epithelium, thickened fibrous lamina and increased inflammatory infiltration $(8,15)$.

Three factors are considered to lead to the development of KCOT in the jaws: i) epithelial proliferation; ii) increased intracystic fluid pressure; iii) molecules associated with bone resorption, such as IL1, IL6 and PGE2 (16).

A series of biomarkers that are highly expressed in KCOTs prior to decompression were previously reported to exhibit a marked decrease following therapy, such as IL- $1 \alpha$-induced PGs and collagenase (8), Ki-67 (8,18), Bcl-2 (17) and KGF (19), indicating the attenuation of cell proliferation, anti-apoptosis and local invasion of the KCOT epithelial cells. These changes may be attributed to the release of intracystic fluid pressure and the communication with the oral environment. However, the precise mechanism has not been fully elucidated.

Among the various biological markers involved in KCOT, the number of available studies using an anti-COX-2 antibody for the investigation of odontogenic tumours is limited. Mendes et al (7) reported a positive COX-2 expression in all the KCOT samples included in their study, with mild to strong 
Table I. Clinical information and intensity of cyclooxygenase-2 (COX-2) expression.

\begin{tabular}{|c|c|c|c|c|c|c|}
\hline \multirow[b]{2}{*}{ Case no. } & \multirow{2}{*}{$\begin{array}{l}\text { Age } \\
\text { (years) }\end{array}$} & \multirow{2}{*}{$\begin{array}{l}\text { Gender } \\
(\mathrm{M} / \mathrm{F})\end{array}$} & \multirow{2}{*}{$\begin{array}{l}\text { Duration of } \\
\text { decompression } \\
\text { (months) }\end{array}$} & \multirow[b]{2}{*}{ Location } & \multicolumn{2}{|c|}{$\begin{array}{l}\text { Outcome (expression } \\
\text { degree of COX-2) }\end{array}$} \\
\hline & & & & & Group I & Group II \\
\hline 1 & 22 & M & 27 & Mandible; Mol-Ram & 1 & 0 \\
\hline 2 & 15 & M & 10 & Mandible; Ang-Ram & 2 & 0 \\
\hline 3 & 20 & M & 21 & Mandible; Ang-Ram & 2 & 0 \\
\hline 4 & 42 & M & 17 & Mandible; Mol-Ram & 0 & 0 \\
\hline 5 & 20 & $\mathrm{~F}$ & 16 & Mandible; Ang-Ram & 1 & 0 \\
\hline 6 & 13 & $\mathrm{~F}$ & 12 & Mandible; Mol-Ram & 2 & 0 \\
\hline 7 & 38 & $\mathrm{~F}$ & 3 & Mandible; Ang-Ram & 1 & 0 \\
\hline 8 & 34 & $\mathrm{~F}$ & 16 & Mandible; Mol-Ram & 2 & 0 \\
\hline 9 & 49 & $\mathrm{~F}$ & 18 & Mandible; Ang-Ram & 1 & 1 \\
\hline 10 & 55 & M & 15 & Maxilla; Ant & 1 & 0 \\
\hline 11 & 25 & $\mathrm{~F}$ & 15 & Mandible; Mol-Ram & 2 & 0 \\
\hline 12 & 33 & $\mathrm{~F}$ & 9 & Mandible; Ang-Ram & 1 & 1 \\
\hline 13 & 35 & M & 23 & Mandible; Ang-Ram & 1 & 1 \\
\hline 14 & 24 & $\mathrm{~F}$ & 31 & Mandible; Ang-Ram & 1 & 0 \\
\hline 15 & 29 & M & 23 & Maxilla; Ant & 1 & 0 \\
\hline 16 & 28 & $\mathrm{~F}$ & 27 & Maxilla; Ant & 2 & 1 \\
\hline
\end{tabular}

Clinical information and intensity of COX-2 expression. Group I, samples obtained at the time of decompression; group II, samples obtained at the time of two-stage enucleation. Immunohistochemical activity: 0, negative; 1, weakly to moderately positive; 2 , strongly positive. M, male; F, female; Mol, molar region; Ang, angular region; Ram, mandibular ramus; Ant, anterior region.
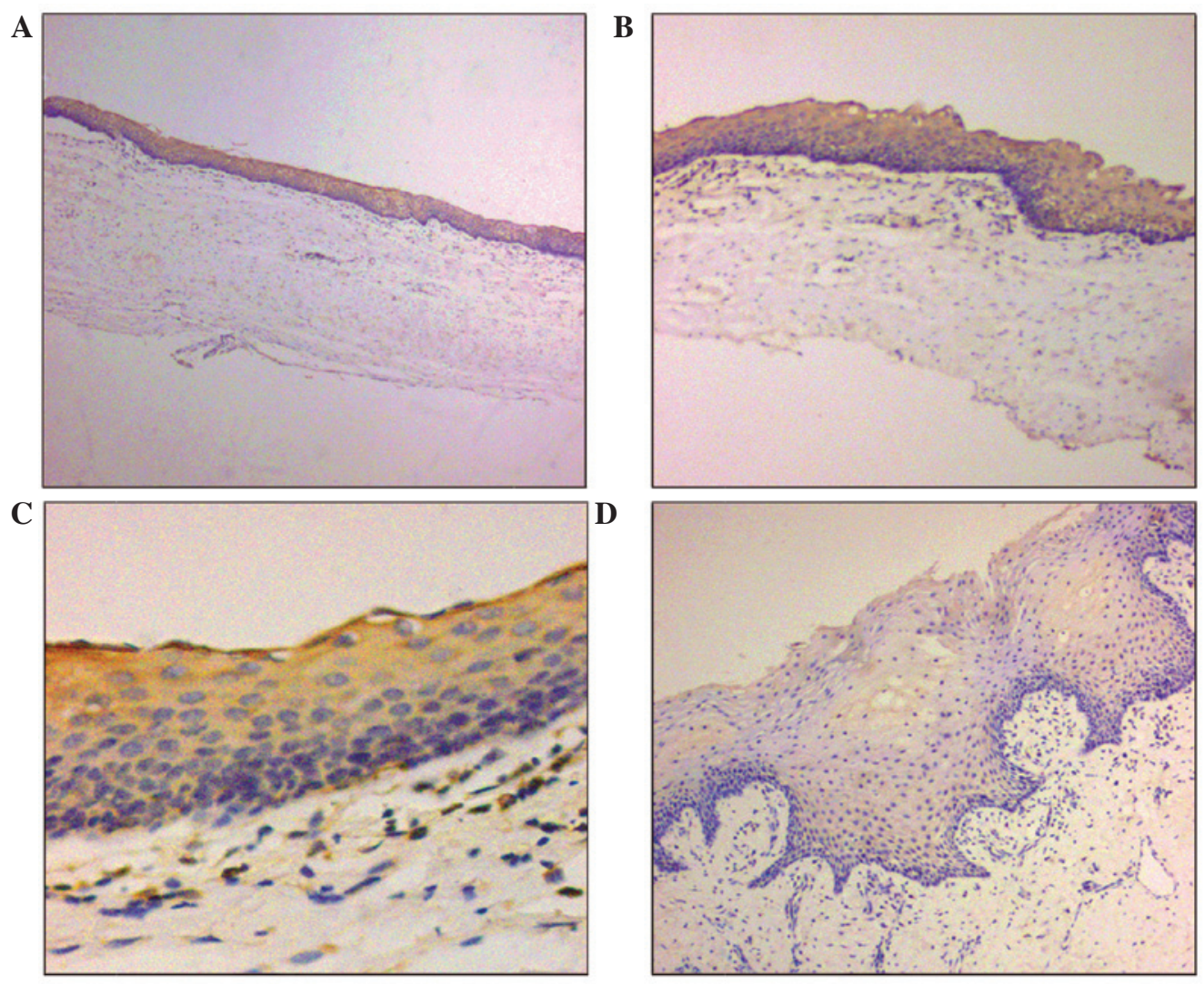

Figure 1. Cyclooxygenase-2 (COX-2) expression in the keratocystic odontogenic tumour (KCOT) epithelium. (A) Sample prior to decompression. Section of positive COX-2 staining in the KCOT epithelium (magnification, x40). (B) Sample prior to decompression. Section of strong COX-2 staining intensity in the KCOT epithelium (magnification, x100). (C) Sample prior to decompression. Positive COX-2 staining involving the full thickness of the KCOT cystic epithelial lining (magnification, x400). (D) Sample following decompression. Section of mild COX-2 staining intensity in the KCOT epithelium (magnification, x100). 
expression levels and a cytoplasmic staining pattern. In the present study, a positive COX-2 expression was observed in 15 of the 16 cases in group I and the immunostaining involved the full thickness of the epithelial lining. This result was consistent with that reported by Mendes et al (7). As regards the staining pattern, the present study demonstrated a cytoplasmic and membranous staining pattern, whereas Mendes et al (7) reported only cytoplasmic staining. At the time of enucleation, only 4 cases exhibited COX-2 expression levels consistent with their corresponding counterparts in group I. In the remaining 12 cases the staining was negative.

To the best of our knowledge, this is the first study on the differentiation of COX-2 expression in KCOTs between one-stage decompression and two-stage enucleation. However, the exact mechanism of COX-2 involvement in the pathogenesis and progression of KCOT has not been elucidated.

Cyclooxygenase is a key regulatory enzyme involved in the conversion of arachidonic acid to PGs (10). As one of the isoforms, COX-2 expression is induced under physiopathological conditions, such as inflammatory stimuli and oncogenes (10). By binding to a G protein-coupled surface receptor and leading to changes of intracellular cyclic AMP and calcium (20), PGs play a key role in several important physiological processes such as oncogenesis, inflammation, cell reproduction and apoptosis and bone metabolism (9).

Mechanical stress is crucial in the regulation of bone metabolism (21). According to Kubota et al (22) the intracystic fluid pressure of odontogenic cysts in the jaws is $>80 \mathrm{mmHg}$ during the early growth stage. However, whether the positive intracystic fluid pressure affects the growth of odontogenic jaw cysts such as KCOT and the exact underlying mechanism have not been fully elucidated. Interleukin-1 $\alpha$ (IL-1 $\alpha$ ) is a multifunctional proinflammatory cytokine $(10,18)$. A previous study by Oka et al (18) demonstrated that the positive pressure upregulated the expression of IL-1 $\alpha$ in KCOT epithelial cells. Furthermore, in a co-culture of KCOT fibroblasts and epithelial cells, the IL- $1 \alpha$ secreted by the epithelial cells further induced the secretion of MMP-1, MMP-2, MMP-3, PGE2 and M-CSF by the fibroblasts, which ultimately stimulated osteoclastogenesis and collagen degradation (18). Moreover, it was previously verified that the transcription and expression levels of COX-2 were significantly decreased in KCOT epithelial linings following decompression (8). There appears to be a strong correlation among intracystic pressure, expression of IL- $1 \alpha$ and bone resorption, with regulation of COX-2 and PGE2 being the key link in this process. Apart from the intracystic fluid pressure, mechanical loading on bone generates a fluid flow within the mineralized matrix, which may exert fluid shear stress (FSS) on cell membranes (23). A previous study by Wadhwa et al (23) reported that FSS may induce the transcription of COX-2 in MC3T3-E1 osteoblasts through the PKA and ERK signaling pathways.

The findings mentioned above may explain the possible mechanism underlying the mechanical stress-dependent growth of KCOT in the jaws. Following decompression, the release of fluid pressure leads to the gradual reduction of bone resorption (18) and shrinking of the tumour size (8), which is at least partially mediated by the COX-2/PGE2 regulation.

In conclusion, despite the small size of this sample, our results combined with the current knowledge of the effects of
COX-2 on oncogenesis suggest that COX-2 may contribute to the biological profile of KCOTs by affecting the biological regulation of the tumoural epithelium. In the present study, although a notable change was observed in COX-2 expression following decompression, the potential association between this change and the clinical characteristics of KCOT, such as the duration of decompression, tumour location and recurrence rate, could not be further assessed, due to the small sample size and short follow-up period. These issues require further investigation.

\section{Acknowledgements}

This study was funded by the Priority Academic Program Development of Jiangsu Higher Education Institutions (PAPD).

\section{References}

1. Shear M: The aggressive nature of the odontogenic keratocyst: is it a benign cystic neoplasm? Part 2. Proliferation and genetic studies. Oral Oncol 38: 323-331, 2002.

2. Madras J and Lapointe H: Keratocystic odontogenic tumour: reclassification of the odontogenic keratocyst from cyst to tumour. J Can Dent Assoc 74: 165h-165h, 2008.

3. Bhargava D, Deshpande A and Pogrel MA: Keratocystic odontogenic tumour (KCOT) - a cyst to a tumour. Oral Maxillofac Surg 16: 163-170, 2012.

4. Li TJ, Browne RM, Prime SS, Paterson IC and Matthews JB: p53 expression in odontogenic keratocyst epithelium. J Oral Pathol Med 25: 249-255, 1996.

5. Piattelli A, Fioroni M, Santinelli A and Rubini C: Expression of proliferating cell nuclear antigen in ameloblastomas and odontogenic cysts. Oral Oncol 34: 408-412, 1998.

6. Kim DK, Ahn SG, Kim J and Yoon JH: Comparative Ki-67 expression and apoptosis in the odontogenic keratocyst associated with or without an impacted tooth in addition to unilocular and multilocular varieties. Yonsei Med J 44: 841-846, 2003.

7. Mendes RA, Carvalho JF and van der Waal I: A comparative immunohistochemical analysis of COX-2, p53, and $\mathrm{Ki}-67$ expression in keratocystic odontogenic tumours. Oral Surg Oral Med Oral Pathol Oral Radiol Endod 111: 333-339, 2011.

8. Ninomiya T, Kubota Y, Koji T and Shirasuna K: Marsupialization inhibits interleukin-1 $\alpha$ expression and epithelial cell proliferation in odontogenic keratocysts. J Oral Pathol Med 31: 526-533, 2002.

9. Gallo O, Masini E, Bianchi B, Bruschini L, Paglierani M and Franchi A: Prognostic significance of cyclooxygenase-2 pathway and angiogenesis in head and neck squamous cell carcinoma. Hum Pathol 33: 708-714, 2002.

10. Mendes RA, Carvalho JF and Waal Iv: An overview on the expression of cyclooxygenase- 2 in tumours of the head and neck. Oral Oncol 45: e124-e128, 2009.

11. Suyama Y, Kubota Y, Ninomiya T and Shirasuna K: Immunohistochemical analysis of interleukin-1 $\alpha$, its type I receptor and antagonist in keratocystic odontogenic tumours. J Oral Pathol Med 37: 560-564, 2008.

12. Lin DT, Subbaramaiah K, Shah JP, Dannenberg AJ and Boyle JO: Cyclooxygenase-2: a novel molecular target for the prevention and treatment of head and neck cancer. Head Neck 24: 792-799, 2002.

13. Williams CS, Tsujii M, Reese J, Dey SK and DuBois RN: Host cyclooxygenase- 2 modulates carcinoma growth. J Clin Invest 105: 1589-1594, 2000.

14. Shudou H, Sasaki M, Yamashiro T, Tsunomachi S, Takenoshita Y, Kubota Y, Ninomiya T, Kawazu T and Mori Y: Marsupialisation for keratocystic odontogenic tumours in the mandible: longitudinal image analysis of tumour size using 3D visualised CT scans. Int J Oral Maxillofac Surg 41: 290-296, 2012.

15. Nakamura N, Mitsuyasu T, Mitsuyasu Y, Taketomi T, Higuchi Y and Ohishi M: Marsupialization for odontogenic keratocysts: long-term follow-up analysis of the effects and changes in growth characteristics. Oral Surg Oral Med Oral Pathol Oral Radiol Endod 94: 543-553, 2002.

16. August M, Faquin WC, Troulis MJ and Kaban LB: Differentiation of odontogenic keratocyst epithelium after cyst decompression. J Oral Maxillofac Surg 61: 678-683, 2003. 
17. Pogrel MA and Jordan RC: Marsupialization as a definitive treatment for the odontogenic keratocyst. J Oral Maxillofac Surg 62: 651-655, 2004

18. Oka S, Kubota Y, Yamashiro T, Ogata S, Ninomiya T, Ito S and Shirasuna K: Effects of positive pressure in odontogenic keratocysts. J Dent Res 84: 913-918, 2005.

19. Suyama Y, Kubota Y, Yamashiro T, Ninomiya T, Koji T and Shirasuna K: Expression of keratinocyte growth factor and its receptor in odontogenic keratocysts. J Oral Pathol Med 38: 476-480, 2009.

20. Mohan S and Epstein JB: Carcinogenesis and cyclooxygenase: the potential role of COX-2 inhibition in upper aerodigestive tract cancer. Oral Oncol 39: 537-546, 2003.
21. Kanzaki H, Chiba M, Shimizu Y and Mitani H: Periodontal ligament cells under mechanical stress induce osteoclastogenesis by receptor activator of nuclear factor $\mathrm{\kappa B}$ ligand up-regulation via prostaglandin E2 synthesis. J Bone Miner Res 17: 210-220, 2002.

22. Kubota Y, Ninomiya T and Oka S: Interleukin-1 $\alpha$-dependent regulation of matrix metalloproteinase-9(MMP-9) secretion and activation in the epithelial cells of odontogenic jaw cysts. J Dent Res 79: 1423-1430, 2000.

23. Wadhwa S, Choudhary S, Voznesensky M, Epstein M, Raisz L and Pilbeam C: Fluid flow induces COX-2 expression in MC3T3-E1 osteoblasts via a PKA signaling pathway. Biochem Biophys Res Commun 297: 46-51, 2002. 